Laboratorio de Arte,4-1991 http://dx.doi.org/10.12795/LA.1991.i04.10

\title{
LA CUSTODIA PROCESIONAL DE LA PARROQUIA DE SAN PEDRO DE CARMONA: UNA OBRA DEL SIGLO XVIII
}

\author{
por María Jesús Mejías Alvarez
}

\begin{abstract}
Tras el análisis y descripción de la custodia procesional de la parroquia de San Pedro de Carmona se llega a la conclusión de que se trata de una obra realizada en la primera mitad del siglo XVIII por los plateros carmonenses Antonio de Luna y Francisco de Luna, saliendo así del error de décadas que la establecían como pieza de fines del siglo XVI. Aportamos, además, la documentación necesaria hallada en el Archivo Parroquial de San Pedro que acredita la autoría y la ejecución de la obra.

From the analysis and description of a processional custodial from the church of San Pedro in Carmona, it is concluded that the piece was made in the first half of the 18 th century by the Carmona silversmiths Antonio de Luna and Francisco de Luna, and not, as was thought for decades, at the end of the 16th century. This is confirmed by documents found in the Parish Archive of San Pedro recording the authorship and execution of the work.
\end{abstract}

La Fiesta en honor a la Eucaristía, Corpus Christi, fue promovida a partir de 1193 por la beata Juliana de Mont-Comillon, y aprobada por el obispo de Lieja en 1246. En 1264, el 8 de septiembre, Urbano IV, con la Bula TRANSITURUS, extendió la fiesta a toda la iglesia y fijó su celebración para el jueves siguiente a la octava de Pentecostés. En 1315, Juan XXII estimula la Fiesta añadiendo el mandamiento expreso de llevar en procesión al Santísimo Sacramento, y de aquí arranca el auge que alcanzaron las custodias procesionales en los países cristianos.

En un principio se utilizaban como custodias simples cajas de materiales 
nobles semejantes a los recipientes en los que se guardaban las reliquias. Poco a poco estas cajas fueron mostrando su interior, creándose así la custodia portátil u ostensorio. Las primeras custodias de asiento surgen en la segunda mitad del siglo XIV (custodia turriforme de Ibiza, de plata y esmaltes), y totalmente entrelazadas con la Arquitectura y los estilos arquitectónicos, hecho que se mantendrá hasta la época contemporánea.

Nos detendremos en el modelo renacentista de custodia por ser este al que se ajusta la de San Pedro que a continuación estudiaremos, y por ser el que se mantendrá preferentemente hasta nuestros días con los lógicos cambios en el ornato. Este modelo está compuesto de tres o cinco cuerpos, según el tamaño, pero lo más habitual es que suelan tener sólo tres. Lo más interesante de las custodias renacentistas son sus programas iconográficos cargados de un sentido simbólico y docente. El tema central suele ser el de la Salvación, y para llevar a cabo el proyecto se coordina el Antiguo y Nuevo Testamento.

La custodia de la iglesia parroquial de San Pedro (figura 1), aunque participa de la concepción estructural del modelo renacentista de custodias, no presenta programa iconográfico alguno. Además no transmite con plenitud la mesura y armonía de las obras puramente renacentistas, aunque, sin duda, se trata de un intento bastante cercano a conseguirlo, pues no en balde hasta ahora los estudiosos del tema la habían considerado una obra del siglo XVI, cercana al taller de Francisco de Alfaro.

La custodia de la parroquia de San Pedro de Carmona se compone de peana y tres cuerpos. El primero, de planta cuadrada con salientes laterales que surgen directamente de las esquinas, mide $62 \mathrm{~cm}$. de altura y está destinado a guardar la Sagrada Forma. A cada ochavo saliente le corresponden dos columnas jónicas acanaladas, excepto el tercio inferior, donde nos encontramos un trabajo de cincelado con decoración floral y de óvalos horizontales. Estas soportan un entablamento coronado con frontones rectos sobre los que se apoyan dos elementos de pequeñas dimensiones que parecen ser dos basamentos sin ninguna funcionalidad en el conjunto estético de la obra, quizás estuvieran pensados para apoyar alguna figura de bulto redondo al estilo de las que vemos en la custodia de la Prioral de Santa M. ${ }^{\text {a }}$ de esta misma ciudad de Carmona. Los otros cuatro lados del polígono presentan arcos de medio punto.

La base de este primer cuerpo se ha ocupado con una decoración a base de hojarasca y óvalos horizontales.

En el segundo cuerpo, de menores proporciones y presentando una altura de $48 \mathrm{~cm}$., es de estructura adintelada siguiendo la misma disposición en ochavos del primero. Los intercolumnios están ocupados por pedestales y hornacinas. Las columnas estriadas, con capiteles de orden compuesto, soportan un entablamento, y sobre éste, frontones partidos y enrollados. En este cuerpo intermedio nos hallamos con una figura de bulto redondo de la Fe (figura 2). 
En el basamento de este segundo cuerpo se reproduce el mismo tipo de decoración a base de hojarasca que veíamos en la base del primero.

El tercer y último cuerpo es un templete circular, al estilo del Templo de San Pedro in Montorio (1503, Roma) de Bramante, de $30 \mathrm{~cm}$. de altura. Rodeado de columnas entorchadas salvo el tercio inferior que se nos muestra vacío de decoración, y de capiteles corintios, se cubre con cúpula gallonada que sirve de sustento a la figura que corona toda la custodia, un Cristo Resucitado de $36 \mathrm{~cm}$. de altura de bulto redondo.

La peana sobre la que descansa toda la custodia presenta la misma planta cuadrada con salientes chaflanes que veíamos en los dos primeros cuerpos. Mide $22 \mathrm{~cm}$. de altura, y está formada por molduras escalonadas de perfiles rectos con decoración floral semejante a la del basamento del primer y segundo cuerpos, de gallones en una de sus molduras salientes, y de motivos geométricos.

Si atendemos a su concepción estructural y compositiva nos encontramos con una custodia modelo renacentista, pero esto no es suficiente para catalogarla y fecharla en el último tercio del siglo XVI. Sin lugar a dudas, su estructura es similar a la de las custodias renacentistas obras del maestro platero Francisco de Alfaro, como son la custodia de la iglesia parroquial de San Juan de Marchena (1586), la de la iglesia parroquial de Santa Cruz de Ecija (1578) y, sobre todo, la custodia de la de la iglesia prioral de Santa María de Carmona (1584), hecho que llevó a Hernández Díaz, Sancho Corbacho y Collantes de Terán, en su Catálogo Arqueológico y Artístico de la provincia de Sevilla, a datarla como "obra probable del platero sevillano Francisco de Alfaro, último cuarto del XVI"!

Las obras posteriores ${ }^{2}$ que nos hablan de esta custodia siguen las mismas pautas de datación, que las llevan a continuar con el mismo error cometido en el citado catálogo.

Si nos detenemos en su estudio compositivo y ornamental encontramos en esta obra algunas contradicciones, pues aún siguiendo modelos de custodias de asiento del siglo XVI, no llega a cumplir el ideal renacentista que combina la medida, proporción y equilibrio.

Tras su análisis ornamental ṇos surgieron las primeras dudas sobre si se trataba de una pieza auténtica de fines del siglo XVI, tanto por la calidad de su trabajo de cincelado como por los propios motivos decorativos (figura 3 ). Como

1. HERNÁNDEZ dÍAZ, J.; SANCHO CORBACHO, A., y COLLANTES DE TERÁN, F.: Catálogo Arqueológico y Artístico de la Provincia de Sevilla. Tomo II, p. 157. Sevilla, 1949-1955.

2. CARL HERNMARCK: Custodias Procesionales en España. Ministerio de Cultura, 1987, p. 174, se data la obra "de aproximadamente de 1600 ".

Varios autores: Guia Artística de Sevilla y su provincia. Diputación de Sevilla, 1981, p. 372, nos clasifica la obra como de fines del siglo XVI y vinculable a Francisco de Alfaro.

El Arte en el Corpus. Las Custodias. Caja de Ahorros Provincial San Femando de Sevilla, 1985. Establece la obra como de fines del siglo XVI. 
ya dijimos, la base del primer y segundo cuerpo presenta una decoración a base de hojarasca y óvalos horizontales que aún queriendo recordar a los grutescos, candelieri y óvalos renacentistas no se logran con la misma concepción estética. Otro aspecto en lo decorativo que nos desconcertó fueron las flores tardobarrocas que aparecen en aquellos elementos extraños que veíamos sobre los frontones rectos del primer cuerpo de los que hablábamos al describir la custodia, y que igualmente aparecen en el basamento del segundo cuerpo, así como en las enjutas de los arcos del primer cuerpo (figura 4). Así pues, no nos atrevíamos a refutar la tradicional datación con estas pequeñas contradicciones en el ornato de la pieza que bien pudieran haber sido efecto de retoques posteriores, y con la única base de la intuición de que aunque se trataba de una pieza bien estructurada y pensada con estética renacentista no llegaba a transmitir el equilibrio, la mesura y la armonía de las obras puramente emanadas de ella.

Pero tras llevar a cabo las investigaciones oportunas en el Archivo Parroquial de la iglesia de San Pedro, nuestras dudas se resolvieron, pues en los Libros de Fábrica de la Hermandad Sacramental ${ }^{3}$ de la citada iglesia hallamos la documentación suficiente como para demostrar que la custodia fue realizada en el siglo XVIII por plateros carmonenses.

En 1711, don Miguel Nieto Narbaes, hermano mayor de la Sacramental, determinó hacer una custodia nueva concertándola con Francisco Cansino, maestro platero de Carmona, examinado en $1685^{4}$. Pero al parecer el trabajo que estaba realizando no convencía a la Hermandad, por lo que se acordó no proseguir la obra, determinando el desbarato de ésta y el empiece de otra nueva por parte de otro platero. Para lo cual obtuvieron licencia del señor don Luis de Salsedo y Azcona, arzobispo de Sevilla, el 28 de agosto de 1729. En virtud de esta licencia don Juan González Flores, hermano mayor de la Hermandad, según el auto de cuentas del año 1731, ajustó con Tomás Guisado, maestro arquitecto, una custodia de madera que sería el diseño de la custodia de plata. Para la realización de ésta se contrataron los servicios de don Antonio de Luna y de don Francisco de Luna, padre e hijo, respectivamente, y maestros plateros de Carmona, examinados en 1698 y en $1741^{5}$.

En 1750 se concluyen los tres cuerpos de la custodia y la figura que lo remata, faltando por forrar de plata la tarimilla que la soporta, y las figuras que

3. Libro de Cuentas de fábrica de la Hermandad del Santísimo Sacramento de San Pedro S/N. 1719-1750.

Libro de Cuentas de fábrica de la Hermandad del Santísimo Sacramento de San Pedro n. ${ }^{\circ} 6$. Auto de Cuentas de septiembre de 1750 a julio de 1753. Fol. 17v. Auto de Cuentas de julio de 1757 a 1759; folios 21, 22, 23 r.v.

4. GESTOSO, J.: Ensayo de un diccionario de los Artífices que florecieron en Sevilla desde el siglo XIII al XVIII. 3 tomos: 1899, 1900, 1908. Tomo III, p. 135.

5. Datos extraídos del Archivo Municipal de Carmona. Unidad de Conservación 678, años 1731-1750, y unidad de conservación 676, años 1619-1710. 
se quisieran agregar para un mayor exorno y que nunca se le hicieron, presentándose hoy desnuda de tales figuras. Con la conclusión de la tarimilla en 1759 queda completada la custodia procesional que vemos hoy en la iglesia parroquial de San Pedro de Carmona.

Los tres cuerpos de la custodia a su término pesaron 1869 onzas y 15 adarmes que costaron 29.288 reales y 18 maravedís, mientras que los costes de las hechuras realizadas por los plateros más arriba citados supusieron 13.280 reales y 26 maravedís, lo que hace un total de 42.569 reales y 12 maravedís. A esto hay que agregar las 362 onzas y 14 adarmes que pesó la plata de la tarimilla que realizó Francisco de Luna, cobrando 2.561 reales y 28 maravedís por su trabajo, y el coste de la custodia de madera realizada por Tomás Guisado a precio de 1.270 reales de vellón y que sirvió de diseño para la de plata.

A continuación transcribiremos los documentos que nos han servido para demostrar que la custodia procesional de la parroquia de San Pedro de Carmona es una obra realizada en pleno siglo XVIII, aunque bajo concepciones renacentistas, pues en realidad lo que sus diseñadores pretendieron fue hacer una imitación, aunque simplificada, de la custodia de Francisco de Alfaro, fines del siglo XVI, que se halla en la iglesia prioral de Carmona. 


\section{APÉNDICE DOCUMENTAL}

\section{DOCUMENTO N.․ 1}

\section{9-1750. Carmona \\ Libro de cuentas de fábrica de la REAL Y FERVOROSA HERMANDAD DEL SANTÍSIMO SACRAMENTO de la Iglesia parroquial de San Pedro de Carmona}

Archivo Parroquial de la iglesia de San Pedro. Libro S/n

"Quentas que se le han tomado a los herederos don Juan Gonzalez Flores difunto ermano maior que fue de la cofradia de el S.S. de San Pedro, cargo de tres años hasta fines de 1731:

Custodia de madera

Iten un mill dozientos y setenta reales de vellon que a costado el esqueleto de madera para la custodia de plata que se a de labrar. Se abonan.

Echuras que se le an pagado al platero

Iten quatro mill y quinientos reales que el dicho don Juan Gonzales entrego a Antonio de Luna platero para en quenta de las echuras de dicha custodia (...).

Plata comprada y entrega-

Iten ciento y quarenta y quatro onsas de plata que sean comprado a dida a el platero para la cusferentes personas a diferentes personas las 48 a dies de plata $=471 / 2$ todia onzas a nuebe de plata $=14$ a ocho y medio de plata y las $341 / 2$ restantes a ocho de plata que todas importan dos mill quatrozientos y cuarenta y dos reales de vellon que se abonan (...)".

"Auto de cuentas de primero de octubre de 1733 hasta fin de septiembre de 1734:

Combenio de los plateros

En dicho día 10 de maio de 1734 en prezencia de dicho visitador y vicario y su notario, señores Beneficiados y curas desta iglesia y el presente ermano mayor y otros ermanos se hallaron el dicho Antonio de Luna y Francisco de Luna, su hijo y entre hijo y padre se convinieron con consentimiento de dicho visitador y demas hermanos, en que el dicho Francisco de Luna, avia de tomar a su cargo el proseguir labrando la custodia y que la quenta la avia de tener esta cofradia desde este dia en adelante en el dicho Francisco de Luna, no saliendo el dicho Antonio de Luna de la obligacion de fiar a su hijo segun consta de la escriptura que los dos tenian otorgada para labrar dicha custodia (...)".

"Auto de cuentas de 24 de junio de 1742 hasta septiembre de 1750:

Plata comprada por el presente ermano mayor para rematar el tercer cuerpo
Don Domingo Garzia Franco hermano mayor queda esta quenta en el tiempo della de comprada por mano de don Francisco de Luna maestro platero que a labrado y esta labrando la custodia de plata, 192 onsas y 15 adarmes de plata, con que remato de labrar el tersero 
cuerpo de la custodia las que costaron 3.108 reales y seis maravedis como consta de resibo de dicho Francisco de Luna con fecha de 4 de agosto de 1745.

Iten da pagados el dicho hermano maior en el tiempo de esta quenta dos mil y ochenta y un reales y 32 maravedis de vellon a don Francisco de Luna maestro platero de las hechuras de las 192 onsas y 15 adarmes de plata que labro con que remato el tersero y ultimo cuerpo de la custodia y a presio de 30 reales de plata cada uno, y mas 638 reales que se le quedaron deviendo por esta cofradia como consta de la antecedente quenta, se incluien 81 reales y 8 maravedis que llebo dicho platero por el mayor costo de la hechura del señor de la Asension que tiene por remate dicha custodia.

Se abonaron los tres cuerpos de la custodia de plata que se esta labrando y tiene 13.869 onsas y 15 adarmes y an costado 42.569 reales y 12 maravedis de vellon. Los 292.888 reales y 18 maravedis de ellos, del balor de dichas onsas de plata y los 13.280 reales y 28 maravedis restantes, importante de las hechuras y a prezio de 30 reales de plata de a 16 quartos el marco, y mas 81 reales y 8 maravedis que el platero por el mayor costo de la figura del señor de la Asension que tiene por remate dicha custodia que con expresion es en la forma siguiente:

Aviendo visto y reconosido las quentas que an dado los hermanos mayores que fueron desta cofradia de las rentas dellas sea hallado en las del año de 1711 dadas por don Miguel Nieto de Narbaes, familiar que fue del Santo Oficio de la Inquisicion, que poe esta hermandad y el suso dicho en su nombre se determino el hazer una custodia de plata la que se ajusto con FRANCISCO CANSINO maestro platero que fue desta ciudad a presio de sesenta reales de vellon cada marco de ocho onzas.

Don Miguel Nieto para dar principio a labrar dicha custodia compro y entrego a dicho platero 600 onsas de plata las que costaron 801 reales de vellon y de ellas pago las hechuras a dicho platero, don Francisco de Ojeda por sus quentas que dio el año 1719 consta que el suso dicho compro y entrego a dicho platero $3281 / 2$ omsas de plata que cosstaron 4.882 reales de vellon y pago las hechuras a dicho platero.

$\mathrm{Y}$ estando en este estado la custodia se suspendio el proseguirla por desirse por maestros de plateros, los mas inteligentes, que estaba errada por mala disposizion que le avia dado el maestro platero y que por esta razon no se podia proseguir.

Por las quentas que dieron los erederos de don Juan Gonzalez Flores, ermano mayor desta cofradia en el año de 1731 consta y parese que la hermandad y el suso dicho de conformidad determinaron el desbarato de dicha custodia vieja y que se hisiese una nueba para lo que octuvieron lisensia del señor don Luis de Salsedo y Azcona arzobispo de la ciudad de Sevilla firmada de su mano su fecha en 28 de agosto de 1729.

En virtud de dicha lisensia el dicho don Juan Gonzalez Flores ajusto con Thomas Guisado maestro arquitecto una custodia de madera para que sirbiese de diseño para hazer la de plata nueba en presio de 1270 reales de vellon la que se hizo y pago dicho Juan Gonzalez.

El dicho don Juan Gonzales Flores ajusto la plata, por lo perteneciente a la hechura con Antonio y Francisco de Luna, padre e hijo, maestros plateros en esta ciudad, a presio de 30 reales de plata (de a 16 quartos) cada marco de ocho onsas, se les entrego dicha custodia de madera y la plata que tenia, la de plata vieja (...).

Plata que tienen los tres cuerpos de la custodia en este año de 1750:

- Don Miguel Nieto y don Francisco de Ojeda compraron para hazer la custodia que para hazer esta nueba sea desbaratado y fundido los $9021 / 2$ onsas, que en el presente peso de ellos, se hallo las 
que costaron 12.982 reales como queda expresado y consta de las sitadas quentas de los suso dichos en los años 1711 y 1719 .

- Don Juan Gonzales Flores que compro en su tiempo como consta de sus quentas del año de 1731 144 onsas y le costaron 2.442 reales.

- Don Diego Gonzales Flores compro 450 1/2 onsas de plata como consta de sus quentas de los años desde el de 1732 hasta el de 1737 y costaron 7.696 reales y 12 maravedis.

- Don Caietano Nabarro para rematar y acabar los primeros dos cuerpos de la dicha custodia compro 49 onsas y 5 adarmes y medio y costaron 838 reales y 28 maravedis como consta de sus recibos de 1742 .

- El dicho Caietano Nabarro para empesar el tersero y ultimo cuerpo de la custodia compro 130 onsas y 10 adarmes y medio de plata y le costaron 2.221 reales y 6 maravedis, como consta de la sitada cuenta suso dicho dio en el año de 1742.

- Don Domingo Garzia Franco da compradas para rematar como esta rematado el tersero y ultimo cuerpo de la custodia 192 onsas de plata y quinse adarmes que costaron 3.108 reales y seis maravedis como consta destas quentas.

Pesan y tiene los tres cuerpos de la custodia que estan rematados del todo 1.869 onsas y 16 adarmes de plata y an costado 29.288 reales y 18 maravedis de vellon.

Hechuras pagadas al platero:

- Don Juan Gonzales Flores pago al platero por quenta de dichas hechuras 6.000 reales como consta de sus quentas de 1731.

- Don Diego Gonzales Flores pago al platero por quenta de dichos hechuras 3.962 reales y 30 maravedis como consta de sus quentas desde 1732 a 1737.

- El dicho Caitano Nabarro pago 952 reales y 16 maravedis por quenta de las dichas hechuras y con que acabo de pagar las hechuras de los 1646 onsas y 5 adarmes y medio que pesan los dos primeros cuerpos de la custodia consta de sus quentas del año de 1742 .

- El dicho don Caitano Nabarro pago a dicho platero por quenta de las hechuras del terzero y ultimo cuerpo de la custodia 283 reales y 18 maravedis como consta de sus sitados quentas del año de 1742.

- Don Domingo Garzia Franco queda esta quenta consta de ella a pagado a dicho platero 2.081 reales y 32 maravedis con que acabo de pagarle las hechuras de las 1.869 onsas y 16 adarmes de plata que pesan los tres cuerpos de la custodia rematados de labar por dicho platero del todo y mas 81 reales y 8 maravedis que le pago por el mayor costo de la hechura del señor de la Asension que tiene por remate dicha custodia que todo importa los dichos 2.081 reales y 32 maravedis.

Los tres cuerpos costaron 42.569 reales y 12 maravedis y falta por acabar la tarimilla de ella y mas a costado los 1.270 reales que costo la custodia de madera". 


\title{
DOCUMENTO N. .2
}

\author{
1750-1802. Carmona \\ Libro de cuentas de fábrica de la REAL Y FERVOROSA HERMANDAD DEL SANTÍSIMO \\ SACRAMENTO de la iglesia parroquial de San Pedro de Carmona.
}

Archivo de la Hermandad Sacramental de la iglesia de San Pedro. Libro/6.

"Auto de quentas de septiembre de 1750 a julio de 1753 :

Se está hasiendo una custodia de plata, que tien a cabados enteramente y con toda perfezion, los tres cuerpos della, y le falta, forrar de plata la tarimilla, y mas las figuras de plata que le quieran echar para adorno de dichos tres cuerpos (...)".

"Auto de cuentas de julio de 1757 a julio de 1759:

El presente hermano mayor declara que acabada la tarimilla de plata de la custodia de plata que estaba haziendo esta cofradia, asistio a su peso, y calidad de plata con diferentes hermanos, en casa de don Francisco de Luna maestro platero acuio cargo estaba su hechura y peso trecientas sesenta y dos onsas y catorce adarmes (...).

Las hechuras de las referidas 362 onsas y 14 adarmes de plata que peso la tarimilla de la expresada tarimilla de la custodia, apresio de 30 reales de plata de a 16 quartos el marco de ocho onsas, segun estaba escripturado con dicho platero importaron dos mill quinientos sesenta y un reales y veinte y ocho maravedis (...)". 


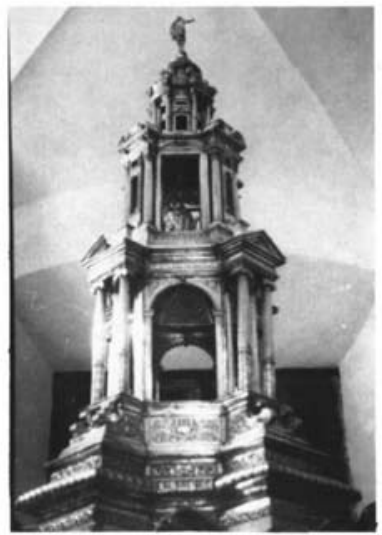

Figura 1

Custodia procesional de la parroquia de San Pedro.

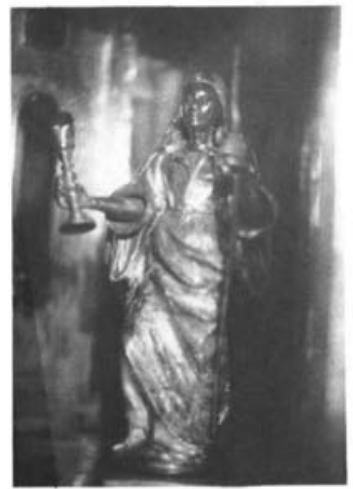

Figura 2

Imagen de la Fe. Detalle de la custodia de San Pedro. 


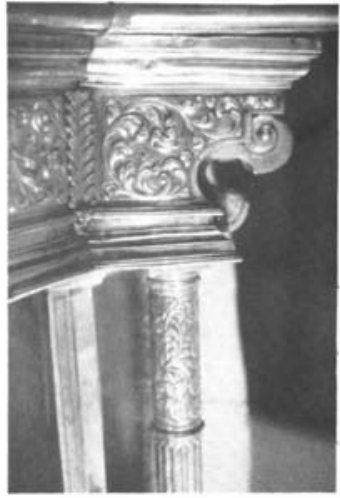

Figura 3

Detalle de la custodia procesional de San Pedro.

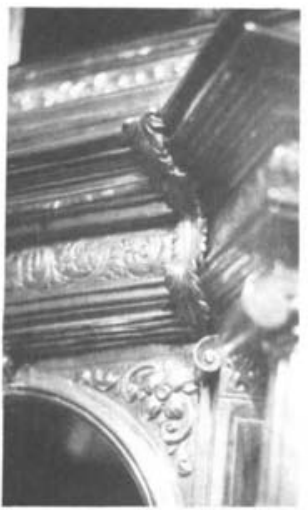

Figura 4

Detalle de la decoración de la custodia de la parroquia de San Pedro. 